\title{
PROGRESS TOWARDS THE CONSTRUCTION OF A TRANSMISSIBLE GASTROENTERITIS CORONAVIRUS SELF-REPLICATING RNA USING A TWO-LAYER EXPRESSION SYSTEM
}

\author{
Zoltán Pénzes, J José Manuel González, Ander Izeta, María Muntión, and \\ Luis Enjuanes \\ Department of Molecular and Cell Biology \\ Centro Nacional de Biotecnología, CSIC \\ Campus Universidad Autónoma \\ Cantoblanco, 28049 Madrid, Spain
}

\section{ABSTRACT}

Three transmissible gastroenteritis coronavirus (TGEV) defective interfering RNAs of $21,10.6$ and $9.7 \mathrm{~kb}$ (DI-A, DI-B and DI-C, respectively) were isolated. Dilution experiments showed that the largest DI RNA, DI-A, is a self-replicating RNA (replicon), and thus codes for a functional RNA polymerase and all the necessary replication signals. In order to engineer a cDNA encoding the RNA replicon a strategy based on the cloning of DI-C cDNA, followed by the insertion of the sequences required to complete the DI-A sequence has been developed. A cDNA complementary to DI-C RNA was cloned under the control of the CMV promoter (pDI-C-CMV) and rescued with a helper virus. In the ORF $1 \mathrm{a}$ of polymerase gene pDI-C-CMV contained a $10 \mathrm{~kb}$ deletion and in ORF $1 \mathrm{~b}$ a $1.1 \mathrm{~kb}$ deletion. The consensus sequence corresponding to the deleted regions was cloned, and the deletions in pDI-C-CMV were replaced to yield a complete cDNA clone of DI-A, pDI-A21-CMV, containing a full-length TGEV polymerase, driven by a CMV promoter. Expression of a functional TGEV polymerase is being investigated.

\footnotetext{
*Z. Pénzes is on sabbatical from the Veterinary Medical Research Institute of the Hungarian Academy of Sciences, Budapest, Hungary.
} 


\section{INTRODUCTION}

Defective RNAs of TGEV PUR46-MAD, DI-A, DI-B, and DI-C of 21, 10.4, and 9.7 $\mathrm{kb}$, respectively were generated by undiluted passages and their structure was determined previously (Méndez et al., 1996), indicating that DI-A RNA contains the information required to encode the viral polymerase, thus it is a potential replicon, similarly to DIssA RNA of MHV which is synthesized in the absence of helper functions (Makino et al., 1988).

In this paper we show that, in fact, DI-A RNA is self-replicating. Our aims were to assemble a full-length cDNA clone of DI-A RNA, and later to study the expression of synthetic DI-A. Since DI-A RNA length is higher than $21 \mathrm{~kb}$, its in vitro expression probably would be difficult, suggesting that the expression of this RNA in the cell nucleus under the CMV promoter could provide a procedure leading to a full length DI-A RNA, unless splicing would occur.

A full-length clone of DI-C $(9.7 \mathrm{~kb})$ was assembled after the CMV promoter (pDIC-CMV) and a synthetic DI-C RNA was rescued and stably passaged, indicating that the intracellular synthesis of DI-A RNA under CMV promoter may be an efficient strategy to rescue a TGEV derived replicon.

\section{MATERIALS AND METHODS}

\subsection{Cells and Virus}

Plaque-cloned TGEV PUR46-MAD (Sánchez et al., 1990) was used. The passage and growth of the virus on swine testis (ST) cells has been described (Méndez et al, 1996).

\subsection{RNA Analysis}

Cytoplasmic RNA was extracted from virus-infected ST cells as described previously (Méndez et al., 1996). Northern hybridizations were performed as described (Sambrook et al., 1989) using a ${ }^{32} \mathrm{P}$-labeled cDNA probe complementary to the 3' end of the TGEV sequence. RNA was quantified on a BioRad phosphorimager.

\subsection{Plasmid Construction}

Standard recombinant DNA techniques were used for the construction of all plasmids. The cDNA clone of $9.7 \mathrm{~kb}$ DI-C RNA behind a T7 promoter, pDI-C (Izeta et al., 1997) was used as a starting point to construct the pDI-A-21. The required fragments of genes $1 \mathrm{a}$ and $1 \mathrm{~b}$ were synthesized by RT-PCR as described (Méndez et al., 1996), cloned and all but one mutation introduced by the PCR were corrected (see Results). pDI-A-21 with a total length of $23.5 \mathrm{~kb}$, was assembled in plasmid pACNR 1180 , kindly provided by J.D. Tratschin (Ruggli et al., 1996). For the construction of plasmids carrying a cDNA encoding DI-C and DI-A under the control of the CMV promoter (pDI-C-CMV and pDI-A21-CMV, respectively), the CMV promoter was amplified from pcDNA3.1 (Invitrogen) and was directly joined to the 5' end of the TGEV sequence by PCR-directed mutagenesis (Dubensky et al., 1996). A cDNA coding for the bovine growth hormone (BGH) terminator sequences was extracted by PCR from plasmid pcDNA3.1 and placed immediately downstream of the cDNAs complementary to DI-C and DI-A RNAs plus a synthetic polyA tract and a hepatitis delta ribozyme (HDVR) to generate perfect 3' ends. 


\subsection{DNA Transfection}

Plasmid DNA was transfected into ST cells using DOTAP liposomal transfection reagent (Boehringer Mannheim) according to the manufacturer's instructions.

\section{RESULTS}

\subsection{Rescue of TGEV Derived Minigenomes Using the CMV Promoter and Cellular Polymerase II}

The use of the CMV promoter for the expression of TGEV minigenomes was studied. pDI-C, a full-length cDNA clone of DI-C RNA containing a T7 promoter, was modified by PCR-mediated site-directed mutagenesis to include the CMV promoter joined precisely to the TGEV 5' end. Accordingly, the 3' end of the DI-C cDNA was engineered to contain the polyA, followed by the HDVR and the BGH terminator sequences. Plasmid DNA was transfected into ST cells and cells were infected with TGEV helper virus. The virus was passaged undiluted and total cellular RNA was analyzed by Northern hybridization in passage 4. DIC RNA was clearly detected (Fig. 1). The expression of the virus containing the DI-C minigenome was stable for more than ten passages (results not shown).

To study the expression of a foreign gene by the TGEV DI vector system, a DI-Cderived minigenome, M39, containing the beta-glucuronidase (GUS) reporter gene under a TGEV subgenomic promoter (Izeta et al., 1997) was constructed and the expression of the minigenome was engineered under the control of CMV promoter. Upon lipofection of the plasmid DNA into ST cells and infection with helper virus, high amounts GUS activity were detected in cell lysates prepared from passage zero, indicating the expression of GUS (data not shown).

Figure 1. Rescue of DI-C RNA expressed from transfected plasmid in the nucleus under the control of the CMV promoter. Northern blot analysis of the RNAs expressed after plasmid pDI-C-CMV was transfected into ST cells and infected with helper virus. Following serial undiluted passages, total cellular RNA was analyzed from passage 4 . Rescue of DI-C RNA was detected as a strong band migrating above the $\mathrm{S}$ mRNA. The left lane shows the standard pattern of mRNAs after infection with the helper virus. The lane on the right side shows the mRNA pattern plus a band with the size expected for the DI-C RNA.

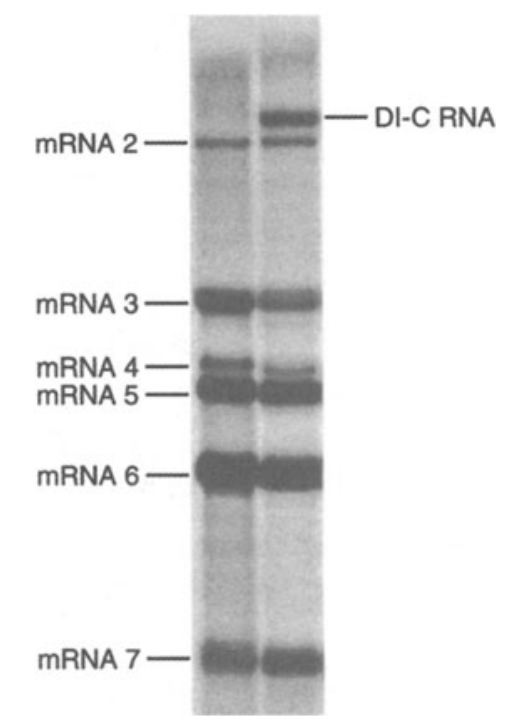




\subsection{TGEV DI-A Minigenome Is a Self-Replicating RNA}

To study TGEV DI-A RNA replication, ST cells were infected with twofold serially diluted DI-containing TGEV. At $7 \mathrm{hr}$ p.i. total cellular RNA was extracted, electrophoresed in a formaldehyde agarose gel, transferred to a nylon membrane by vacuum blotting and hybridized to a ${ }^{32} \mathrm{P}$-labeled cDNA probe complementary to the 3 ' untranslated region. The RNA bands corresponding to the genomic RNA (gRNA), DI-A, DI-B, and the spike (S) mRNA were quantified on a phosphorimager and the graphs were fitted by linear regression analysis. The effects of the virus dilution on the synthesis of the gRNA, DI-A, DI-B, and mRNA S were evaluated (Fig. 2). The levels of gRNA, DI-A and mRNA S decreased linearly with the virus dilution, following one-hit kinetics, while DI-B synthesis decreased more rapidly, following two-hit kinetics. These data indicated that DI-A RNA was synthesized independently of a helper function, while for the replication of DI-B RNA, in addition to the DI-B RNA, a second component was required, most probably the RNA corresponding to the helper virus which encoded the viral replicase.

\subsection{Progress towards the Construction of a Synthetic TGEV Derived Replicon}

The complete cDNA clone of DI-C RNA, pDI-C, (Izeta et al., 1997) was used as a starting point to construct a potential TGEV replicon of $21 \mathrm{~kb}$, that codes for a full TGEV
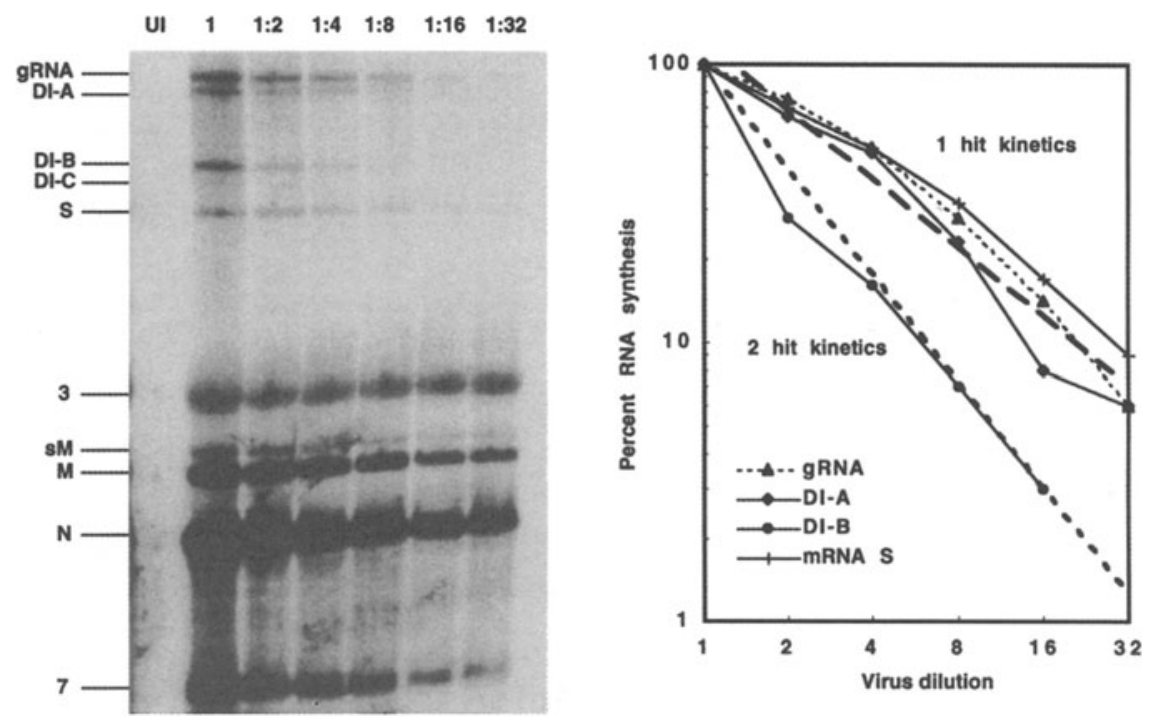

Figure 2. Effects of virus dilution on TGEV RNA synthesis. ST cells were infected with twofold serially diluted DI containing TGEV virus. At $7 \mathrm{hr}$ p.i. total cellular RNA was extracted, electrophoresed on a formaldehyde agarose gel, transferred to a nylon membrane and hybridized to a ${ }^{32} \mathrm{P}$-labeled cDNA probe complementary to the 3 ' untranslated region. The bands corresponding to the gRNA, DI-A, DI-B, and mRNA S were quantified on a phosphorimager and plotted. The theoretical one-hit kinetics and two-hit kinetics are shown as discontinuous lines. The gRNA, DI-A, and mRNA S fit the one-hit kinetics, while DI-B fits the two-hit kinetics curve. 


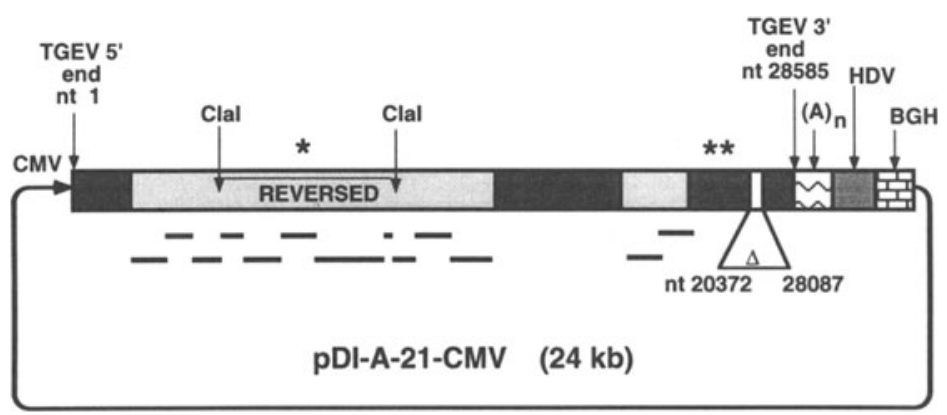

Figure 3. Construction of a TGEV self-replicating RNA cDNA clone. The complete polymerase gene (ORF 1a-b) of TGEV PUR46-MAD and a $0.5 \mathrm{~kb}$ region of the 3 ' end of the genome has been cloned, sequenced and assembled in a single plasmid (pDI-A-21-CMV of $24 \mathrm{~kb}$ ). All errors introduced by the DNA polymerases during cloning have been corrected with the exception of two marker mutations $\left({ }^{*}\right.$, nucleotide 6752 : A to G; ${ }^{* *}$, nucleotide 18997: $T$ to C) which do not change the aminoacids. The dark boxes represent sequences retained from the DI-C plasmid. The light gray boxes represent the filled deletion. The short black lines represent the individual clones used in the construction. (A), polyA; HDV, hepatitis delta ribozyme; $\mathrm{BGH}$, bovine growth hormone terminator; $\Delta$, deleted sequences.

RNA polymerase and also contains sequences from the 5' and 3' ends of the TGEV genome as replication signals. The $10 \mathrm{~kb}$ region of gene $1 \mathrm{a}$, and a $1.1 \mathrm{~kb}$ region in gene $1 \mathrm{~b}$, which were missing from pDI-C, were amplified by RT-PCR in various fragments using a viral RNA preparation of TGEV PUR46-MAD as a template. The PCR fragments were cloned, sequenced and all nucleotide differences encountered between the PUR46MAD sequences and the cDNA clones were corrected with the exception of a silent marker mutation at nucleotide 6752 (A to G, leading to a Ser) (Fig. 3). The complete TGEV replicon cDNA clone was assembled in a low copy number plasmid pACNR 1180 behind CMV promoter. The replicon cDNA clone, named pDI-A-21-CMV (24 kb), and the 13 clones that were used for the filling up of pDI-C are shown in Fig. 3. To delete an unwanted ClaI site at position 18997 a silent $\mathrm{T}$ to $\mathrm{C}$ change has been introduced by PCR-mediated site-directed mutagenesis. This mutation facilitated the assembly of the full-length clone and will allow us to easily distinguish a future infectious clone from the wild type virus.

Plasmid pDI-A-21-CMV contains a $5.2 \mathrm{~kb}$ ClaI gene 1a fragment in the reverse orientation. Due to toxicity problems in bacteria, stable cloning of this fragment in the correct orientation was not possible. To overcome this problem, an in vitro ligation strategy has been developed, which includes the release and in vitro religation of the previously reversed $5.2 \mathrm{~kb}$ fragment in virus orientation and its transformation into mammalian cells. The presence of the correct assembly of the full length clone encoding the replicase has been shown by PCR analysis (results not shown).

To detect the expression of the replicase activity by the engineered cDNA construct, co-transformation with a plasmid encoding a minigenome carrying a reporter gene (GUS) under the control of a TGEV promoter is being performed. GUS activity would be only detected if a functional polymerase is being expressed, that in turn will led to the mRNA encoding the GUS activity. 


\section{DISCUSSION}

In this chapter we have studied the expression of TGEV minigenomes DI-C and M39 containing a foreign gene, GUS, under the nuclear CMV promoter. Rescue of DI-C RNA expressed from CMV promoter was detected when plasmid DNA transfected cells were infected with TGEV helper virus. The rescue of DI-C RNA expressed from CMV promoter was more efficient than the rescue performed with the in vitro transcribed (T7 promoter) and electroporated DI-C RNA (results not shown). This is probably due to the two amplification steps, in which first high amounts of DI-C RNA are transcribed in the nucleus under CMV promoter, followed by a specific amplification by the TGEV polymerase in the cytoplasm. A TGEV minigenome cloned behind CMV promoter was used to express the GUS reporter gene. In the transfected cells (passage 0), the GUS activity was significantly higher within the cells transfected with the DNA construct under CMV promoter control, than when the cells were electroporated with minigenome RNA transcribed in vitro (data not shown). The detection of gene expression in the cells directly transfected with synthetic minigenomes, without the need for amplification throughout the passage in tissue culture, may be a useful tool to study the replication of coronavirus RNA.

For the expression of the generally large synthetic coronavirus RNAs the use of the CMV promoter could be more advantageous than the $\mathrm{T} 7$ promoter, since it would eliminate the need for the in vitro transcription and the manipulation of large synthetic RNAs, and provides a method to synthesize large RNA transcripts in the cells.

The mechanism of synthesis of TGEV DI-A in TGEV infected cells was investigated. Dilution experiments indicated that the synthesis of DI-A RNA followed the same kinetics as gRNA, i.e., the synthesis of DI-A RNA decreased linearly with virus dilution, following one-hit kinetics. Méndez et al. (1996) showed that TGEV DIs are packaged in separate particles. All these data suggest that DI-A was synthesized independently from helper functions, thus codes for a functional polymerase as previously described for DIssA, a large MHV DI RNA that previously has also been shown to replicate independently from helper functions (Makino et al., 1988). In contrast, the synthesis of DI-B RNA decreased exponentially, following two-hit kinetics.

Encouraged by the autoreplication of DI-A RNA, we have constructed a full-length, $21 \mathrm{~kb}, \mathrm{cDNA}$ clone of DI-A RNA by conventional bacterial cloning. Since stable insertion of a $5.2 \mathrm{~kb} \mathrm{ClaI}$ fragment of gene la was not possible in the DI-A context, this fragment was inserted in reverse orientation into pDI-A-21-CMV. Then, in vitro ligation technology was used to release and religate this $5.2 \mathrm{~kb}$ fragment that subsequently was transfected directly into mammalian cells, to prevent the toxicity in bacteria. A cDNA with the correct sequence of the viral replicase has been assembled, as shown by PCR analysis. Current research efforts are being directed to show that the engineered clone encodes a functional replicase.

\section{ACKNOWLEDGMENTS}

This work has been supported by grants from the Consejo Superior de Investigaciones Científicas, the Comisión Interministerial de Ciencia y Tecnología (CICYT), La Consejería de Educación y Cultura de la Comunidad de Madrid, and Fort-Dodge Veterinaria from Spain, and the European Communities (Projects Biotechnology and FAIR). 


\section{REFERENCES}

Dubensky, J., T. W., Driver, D. A., Polo, J. M., Belli, B. A., Latham, E. M., Ibanez, C. E., Chada, S., Brumm, D., Banks, T. A., Mento, S. J., Jolly, D. J., and Chang, S. M. W., 1996, Sindbis virus DNA-based expression vectors: utility for in vitro and in vivo gene transfer, J. Virol. 70: 508-519.

Izeta, A., Smerdou, C., Mendez, A., Alonso, S., Pénzes, Z., and Enjuanes, L., 1997, Replication and packaging of synthetic minigenomes derived from defective transmissible gastroenteritis coronavirus, J. Virol. Manuscript submitted.

Makino, S., Shieh, C. K., Keck, J. G., and Lai, M. M. C., 1988, Defective-interfering particles of murine coronaviruses: mechanism of synthesis of defective viral RNAs, Virology 163: 104-111.

Méndez, A., Smerdou, C., Izeta, A., Gebauer, F., and Enjuanes, L., 1996, Molecular characterization of transmissible gastroenteritis coronavirus defective interfering genomes: Packaging and heterogeneity, Virology 217: 495-507.

Ruggli, N., Tratschin, J. D., Mittelholzer, C., Hofmann, M. A., 1996, Nucleotide sequence of classical swine fever virus strain Alfort/187 and transcription of infectious RNA from stably cloned full-length cDNA, J. Virol. 70: $3478-3487$.

Sambrook, J., Fritsch, E. F., and Maniatis, T., 1989, Molecular cloning: a laboratory manual, Cold Spring Harbor Laboratory, Cold Spring Harbor, New York.

Sánchez, C. M., Jiménez, G., Laviada, M. D., Correa, I., Suñé, C., Bullido, M. J., Gebauer, F., Smerdou, C., Callebaut, P., Escribano, J. M., and Enjuanes, L., 1990, Antigenic homology among coronaviruses related to transmissible gastroenteritis virus, Virology 174: 410-417. 